\title{
E-PSMA: the EANM standardized reporting guidelines v1.0 for PSMA-PET
}

\author{
Francesco Ceci ${ }^{1}$ - Daniela E. Oprea-Lager ${ }^{2}$ (D) - Louise Emmett ${ }^{3,4}$ - Judit A. Adam ${ }^{5}$ - Jamshed Bomanji ${ }^{6}$. \\ Johannes Czernin ${ }^{7}$. Matthias Eiber ${ }^{8}$. Uwe Haberkorn ${ }^{9}$ - Michael S. Hofman ${ }^{10,11}$ • Thomas A. Hope ${ }^{12}$. \\ Rakesh Kumar ${ }^{13}$. Steven P. Rowe ${ }^{14} \cdot$ Sarah M. Schwarzenboeck ${ }^{15}$. Stefano Fanti ${ }^{16} \cdot$ Ken Herrmann $^{17}$
}

Received: 8 January 2021 / Accepted: 7 February 2021 / Published online: 19 February 2021

(C) The Author(s) 2021

\begin{abstract}
Rationale The development of consensus guidelines for interpretation of Prostate-Specific Membrane Antigen (PSMA)-Positron Emission Tomography (PET) is needed to provide more consistent reports in clinical practice. The standardization of PSMA-PET interpretation may also contribute to increasing the data reproducibility within clinical trials. Finally, guidelines in PSMA-PET interpretation are needed to communicate the exact location of findings to referring physicians, to support clinician therapeutic management decisions.

Methods A panel of worldwide experts in PSMA-PET was established. Panelists were selected based on their expertise and publication record in the diagnosis or treatment of $\mathrm{PCa}$, in their involvement in clinical guidelines and according to their expertise in the clinical application of radiolabeled PSMA inhibitors. Panelists were actively involved in all stages of a modified, nonanonymous, Delphi consensus process.

Results According to the findings obtained by modified Delphi consensus process, panelist recommendations were implemented in a structured report for PSMA-PET.

Conclusions The E-PSMA standardized reporting guidelines, a document supported by the European Association of Nuclear Medicine (EANM), provide consensus statements among a panel of experts in PSMA-PET imaging, to develop a structured report for PSMA-PET in prostate cancer and to harmonize diagnostic interpretation criteria.
\end{abstract}

Keywords PSMA-PET $\cdot$ PSMA prostate cancer $\cdot$ Structured report $\cdot$ Consensus panel $\cdot$ EANM guidelines $\cdot$ Prostate cancer guidelines

\section{Introduction}

Prostate-specific membrane antigen (PSMA) is one of the most successful targets for imaging and therapy in nuclear medicine. PSMA is a glycoprotein, a membrane bound metallo-peptidase, encoded by FOLH1 gene on chromosome 11. The protein acts as a glutamate carboxypeptidase on different alternative substrates, including the nutrient folate and the neuropeptide N-acetyl-1-aspartyl-1-glutamate (NAAG) and

This article is part of the Topical Collection on Oncology - Genitourinary

Stefano Fanti and Ken Herrmann are co-senior authors of this project.

Daniela E. Oprea-Lager

d.oprea-lager@amsterdamumc.nl

Extended author information available on the last page of the article is expressed in a number of tissues such as prostate, kidney, and salivary glands [1]. The upregulation of PSMA in prostate cancer (PCa) cells is well known and is used as an effective diagnostic marker for the presence of PCa. This overexpression is present in over $90 \%$ of PCa cells, making PSMA a reliable tissue biomarker for PCa functional imaging [2]. The current hypothesis concerning the function of PSMA is that it plays a role in folate transportation and metabolism. The extra-membrane part of PSMA potentially hydrolyzes glutamated folates released by dying tumor cells. The created folate may be taken up by healthy PCa cells, facilitating further cell proliferation [1]. There is a direct effect of the PSMA receptor on the AkT and PI3K growth pathways, and it likely has a strong role as a driver of cell growth in PCa $[2,3]$. PSMA expression levels increase according to the stage and tumor grade, as well as aneuploidy and biochemical recurrence (BCR). Higher levels of PSMA expression are 
associated with poorer prognostic outcomes [3]. More importantly, PSMA expression is upregulated when castrateresistant phenotype evolves $[4,5]$.

This characteristic makes PSMA particularly attractive since it has potential as an early indicator of progression and tumor heterogeneity in castration-resistant prostate cancer (CRPC) [5]. The localization of the catalytic site of PSMA in the extracellular domain has allowed for the development of very small and highly specific inhibitors that once radiolabeled (namely with ${ }^{68} \mathrm{Ga}$ or

${ }^{18} \mathrm{~F}$ ) are used as radiopharmaceuticals for positron emission tomography (PET) imaging $[6,7]$. This favorable biological and biochemical characteristic of PSMA-based PET/computed tomography (CT) imaging is a key driver among the newgeneration imaging techniques [8-10].

\section{Clinical guidelines and previous PSMA-PET evaluation systems}

Clinical guidelines in PCa have been promoted by several medical societies, namely urological, oncological, and radiation oncology societies. Recently, the European Association of Urology (EAU) guidelines on $\mathrm{PCa}$ [8] recommend the use of PSMA-PET imaging for any case of biochemical recurrence after radical prostatectomy (PSA $>0.2 \mathrm{ng} / \mathrm{mL}$ ), namely if PSMA-PET scan is able to positively influence the subsequent treatment strategy. According to the most recent literature, BCR after primary definitive therapy represents the clinical scenario where PSMA-based imaging has the highest impact on patient management and the clinical decision-making process [11]. In this clinical setting, PSMA-PET provided superior diagnostic accuracy compared to other radiotracers, such as choline or fluciclovine [12, 13]. PSMA-based PET imaging is characterized by a high target-to-background ratio, which results in superior sensitivity and high inter-reader agreement $[12,14]$. Initial staging prior to radical treatment in high-risk PCa or CRPC might be further applied for PSMAPET, even though current clinical guidelines do not yet recommend these. Recently, both ASCO guidelines [15] and EAU consensus conference in management of advanced $\mathrm{PCa}$ [16] promoted the use of new-generation imaging, including PSMA-PET, for investigating advanced PCa. PSMA-PET showed high accuracy to detect PCa lesions in patients with non-metastatic PCa on conventional imaging. Finally, PSMA-PET is a key requisite in later stages of the disease considering the increasing importance of PSMAtargeted radioligand therapy (RLT) [17].

Uniform and reproducible image interpretation is important in providing comparable data between clinical trials and to meet emerging clinical diagnostic needs. While research reporting tools need to be reproducible and accurate to allow for stratification of patient cohorts or to provide the structure for pooling of data, clinical diagnostic reporting tools need to be simple and adaptable to specific clinical situations. Harmonization of PSMA-PET interpretation is also needed to communicate the exact locations of findings to referring physicians, to support clinician therapeutic management decisions, as is the case for metastasis-directed therapy.

Recently, three different criteria were published to improve objectivity and accuracy in image interpretation for PSMA-PET: EANM criteria [18], PROMISE criteria [19], and PSMA-RADS [20]. These three PSMA interpretation criteria were recently compared within an external validation [21], in order to assess the inter-reader, intra-reader, and inter-criteria agreement. The three proposed criteria have good reproducibility in evaluating $\left[{ }^{68} \mathrm{Ga}\right] \mathrm{Ga}$-PSMA-11 PET. However, there are factors leading to inter-reader disagreement indicating that further work is needed to harmonize and/or improve the interpretation criteria for PSMA-PET imaging in order to find the right balance between accuracy and the time requirements for each system.

\section{Radiolabeled PSMA ligands: physiological uptake, variants, diagnostic accuracy, and pitfalls}

\section{PSMA-binding variants}

$\left[{ }^{68} \mathrm{Ga}\right] \mathrm{Ga}-\mathrm{PSMA}-11$ was introduced in 2011 by the German Cancer Research Centre [7]. As the most used radioligand, its success can be explained by the relatively straightforward isotope production (germanium-gallium generator) and radiolabeling of the tracer [22]. $\left[{ }^{18} \mathrm{~F}\right] \mathrm{DCFPyL}[23]$ is another widely used tracer that was introduced as a successor to $\left[{ }^{18} \mathrm{~F}\right] \mathrm{DCFBC}$ [24]. Compared to $\left[{ }^{68} \mathrm{Ga}\right] \mathrm{Ga}$-PSMA- $11,{ }^{18} \mathrm{~F}$-labeled compounds have longer half-life, allowing imaging at later time point, a higher production capacity and a more centralized production $[25,26]$. $\left[{ }^{18} \mathrm{~F}\right] \mathrm{PSMA}-1007$ represents another ${ }^{18} \mathrm{~F}$-tracer variant characterized by predominant hepatobiliary excretion [27], thus reducing the urinary excretion of the radiotracer. Several other PSMA ligands are available for PET imaging (e.g., $\left[{ }^{18} \mathrm{~F}\right] \mathrm{rh}-\mathrm{PSMA}$, $\left[{ }^{68} \mathrm{Ga}\right] \mathrm{Ga}-P S M A$ I\&T, $\left[{ }^{18} \mathrm{~F}\right] \mathrm{JK}-\mathrm{PSMA}-7,\left[{ }^{68} \mathrm{Ga}\right] \mathrm{Ga}-P S M A-$ $\mathrm{R} 2$ ), but few data in literature are available at present $[28,29]$.

\section{Physiological uptake}

For $\left[{ }^{68} \mathrm{Ga}\right] \mathrm{Ga}-\mathrm{PSMA}-11$ and $\left[{ }^{18} \mathrm{~F}\right] \mathrm{DCFPyL}$, high PSMA uptake is noted in the cortex of the kidneys, parotid and submandibular salivary glands, and duodenum. Moderate median uptake $\left(\mathrm{SUV}_{\max }>3\right)$ is noted in the spleen, liver, and lacrimal glands [30]. In comparison to $\left[{ }^{68} \mathrm{Ga}\right] \mathrm{Ga}-\mathrm{PSMA}-11$, the uptake of $\left[{ }^{18} \mathrm{~F}\right] \mathrm{PSMA}-1007$ is higher in the liver, gallbladder, pancreas, and sublingual glands, and lower in the kidneys, bladder, and lacrimal glands [31]. Since daily repeatability on the PSMAligand uptake for each organ is essential for semi-quantitative analysis, knowledge on dosimetry and physiological uptake is 
important [32]. Moreover, knowledge about uptake in benign tissues is important, by enabling to calculate the thresholds that can classify potential malignant lesions when interpreting PETscans [32]. Kidney, spleen, and salivary uptake are higher on $\left[{ }^{68} \mathrm{Ga}\right] \mathrm{Ga}$-PSMA-11 compared to $\left[{ }^{18} \mathrm{~F}\right] \mathrm{DCFPyL}$, while the liver shows slightly lower uptake. The blood pool (aorta) is similar in both $\left[{ }^{18} \mathrm{~F}\right] \mathrm{DCFPyL}$ and $\left[{ }^{68} \mathrm{Ga}\right] \mathrm{Ga}-\mathrm{PSMA}-11$ and can therefore be used as a benchmark in assessing lesions based on $\mathrm{SUV}_{\max }$ [32]. One limitation of both $\left[{ }^{68} \mathrm{Ga}\right] \mathrm{Ga}$-PSMA-11 and $\left[{ }^{18} \mathrm{~F}\right] \mathrm{DCFPyL}$ is their increased urinary excretion in the ureters and the bladder, which limits detection of local recurrence in the prostate bed. This excretion is absent for $\left[{ }^{18} \mathrm{~F}\right] \mathrm{PSMA}-1007$ due to hepatobiliary clearance, and might improve local detection of $\mathrm{PCa}[31]$.

\section{Pitfalls}

The expression of PSMA can predominantly be found in PCa, but benign and other malignant tissues are known to express PSMA and have extensively been described [33]. Here we present an array of pitfalls that can influence reader's interpretations. Although the positive predictive value and specificity of PSMAPET are known to be high [14, 34], cautious reading and knowledge of common pitfalls should be considered while interpreting PSMA-PET images and drafting the medical report.

\section{Other malignancies}

While epithelial PSMA expression is the mechanism for detecting $\mathrm{PCa}$, for other neoplasms, it is hypothesized to be associated with tumor neovasculature [35]. Renal cell carcinoma (RCC) is most commonly described, especially in clear cell RCC [36]. Although it may present as a pitfall, it also creates the opportunity to utilize PSMA-PET in the detection of these malignancies, including RCC [36], hepatocellular carcinoma [37], breast cancer, and lung cancer [38] and other malignancies. These indications are strictly restricted to dedicated research protocols and do not have clinical use yet.

\section{Ganglia}

Ganglia might be considered one of the most common pitfalls, since they can mimic lymph nodes. In both $\left[{ }^{18} \mathrm{~F}\right] \mathrm{DCFPyL}$ and $\left[{ }^{68} \mathrm{Ga}\right]$ Ga-PSMA-11, PSMA expression was observed in at least one ganglion (96.9-98.5\% of all scans) [39]. Since ganglia can mimic the anatomical locations of lymph nodes, knowledge of their presence is important for accurate staging, thus preventing false interpretation. Ganglia should be distinguished from lymph node metastases based on location, tracer uptake, and configuration. Most important for their differential diagnosis, ganglia almost never have a nodular appearance (2.0\%) such as metastatic lymph nodes (58.6\%), and are usually linear shaped (71.2\%) [39].

\section{Benign bone disease}

Most common pitfalls when differentiating bone metastases (M1b) from other pathologies are healing bone fractures and degenerative bone changes $[40,41]$. $\left[{ }^{18} \mathrm{~F}\right]$ PSMA-1007 has been described as expressing increased PSMA expression not only in benign bone lesions compared to $\left[{ }^{68} \mathrm{Ga}\right] \mathrm{Ga}$ PSMA-11, specifically fractures, but also in non-traumarelated PSMA uptake [41]. Several case reports have been published for $\left[{ }^{18} \mathrm{~F}\right] \mathrm{DCFPyL}$ and $\left[{ }^{68} \mathrm{Ga}\right] \mathrm{Ga}$-PSMA-11, showing Paget's disease localizations in the pelvis, humerus, and sacrum [42]. Other reasons reported in the literature are fibroosseous lesions (e.g., fibrous dysplasia and hemangiomas), which are most commonly present in the ribs [43].

\section{Benign neurogenic tumors}

Brain parenchyma normally does not contribute to PSMA uptake, therefore giving the clinician excellent visualization of potential PSMA expressing metastases. However, there have been several case reports of pitfalls in $\left[{ }^{68} \mathrm{Ga}\right] \mathrm{Ga}$ PSMA-11 scans, with PSMA expression seen after ischemic strokes [44]. In addition, gliomas, meningiomas, paragangliomas, and neurofibromas have been described to have PSMA uptake $[45,46]$. Focal uptake in the brain parenchyma on PSMA-PET generally requires MRI correlation.

\section{Pulmonary sarcoidosis/granulomatosis}

Although the uptake mechanisms are not understood, several case reports have described PSMA-ligand uptake in pulmonary sarcoidosis [47]. Another chronic granulomatosis inflammatory disorder that is known to express PSMA is Wegener's granulomatosis [48]. Other inflammatory lung conditions that are known to express PSMA in $\left[{ }^{68} \mathrm{Ga}\right] \mathrm{Ga}$-PSMA-11 scans are bronchiectasis, anthracosilicosis, and tuberculosis [49-51].

\section{Androgen activity}

Androgen receptor (AR) inhibition is believed to increase PSMA expression in $\mathrm{PCa}[4,5]$. This upregulation and its exact timing are not completely understood but must be considered to prevent falsely defining disease progression shortly after initiation of AR-targeted therapies. This increase in PSMA uptake appears transient, since it is more visible during the first weeks of hormonal blockade, with a tendency to decrease over time [5, 52]. However, PSMA upregulation may not be transient, but PSMA-ligand uptake goes down due to treatment response rather than downregulation of PSMA ligand.

Gynecomastia shows increased PSMA uptake and might be observed in patients undergoing ADT [53]. 


\section{Decreased PSMA expression}

Although most pitfalls represent potential false-positive findings, some factors may induce potential false-negative PSMA findings, as well. Up to $5 \%$ of prostate adenocarcinomas do not express PSMA. Furthermore, aggressive forms of primary neuroendocrine $\mathrm{PCa}$ or neuroendocrine de-differentiation after ARtargeted therapies in metastatic CRPC (mCRPC) might show a reduced PSMA expression in metastatic sites [54].

\section{Methodology: the consensus panel}

\section{Rationale of the study}

The development of consensus guidelines for interpretation of PSMA-PET may contribute to provide more consistent reports in clinical practice. The standardization of PSMA-PET interpretation may also contribute to increasing the data reproducibility within clinical trials. Defined criteria for interpreting PSMA-PET images would help improving accuracy, precision, and repeatability of this diagnostic procedure, thus improving patients' management and outcomes. Therefore, consensus interpretation is necessary to provide comparison between clinical trials and to meet upcoming clinical diagnostic needs. Consensus guidelines in PSMA-PET interpretation are also needed to communicate the exact location of findings to referring physicians, to support clinician therapeutic management decisions, as happens for MDT.

In view of these considerations, the E-PSMA standardized reporting guidelines, a document supported by the European
Association of Nuclear Medicine (EANM), is aimed at providing consensus statements among a panel of experts in PSMAPET imaging, to develop a structured report for PSMA-PET in $\mathrm{PCa}$ and to harmonize diagnostic interpretation criteria.

\section{Panel composition}

According to the abovementioned purposes, a panel of worldwide experts in PSMA-PET was established. Panelists were selected based on their expertise and publication record in the diagnosis or treatment of $\mathrm{PCa}$, in their involvement in clinical guidelines and according to their expertise in the clinical application of radiolabeled PSMA inhibitors. The panelists involved are reported in Table 1.

\section{Modified Delphi consensus process}

Panelists were actively involved in all stages of a modified, non-anonymous, Delphi consensus process, as displayed in Fig. 1. In the first two rounds, panelists identified issues regarding PSMA-PET reporting and made proposals about possible criteria to harmonize and standardize the PSMA-PET reporting process. Finally, panelists gave their inputs to identify which criteria or parameters currently used in research studies might be implemented in clinical reports. According to comments, proposal, and suggestions made by panelists during the first and second round, a questionnaire (Fig. 2) composed by 16 questions has been generated. All panelists were asked to answer the questions as in favor or disagree. The inter-rater agreement was measured for each question using Fleiss' kappa (0 poor agreement; $0.01-0.20$ slight

Table 1 Panel composition

\begin{tabular}{llll}
\hline Name & Role & Institution & Country \\
\hline Judit Adam & EANM Oncology and & Amsterdam UMC, University of Amsterdam & The Netherlands \\
Theranostics committee representative & University of London & UK \\
Francesco Ceci & Panelist & University of Turin & Italy \\
Johannes Czernin & Project coordinator & University of California Los Angeles & USA \\
Matthias Eiber & Panelist & Technical University of Munich & Germany \\
Louise Emmett & Panelist & St. Vincent Hospital, Sydney & Australia \\
Stefano Fanti & Project leader & University of Bologna & Italy \\
Uwe Haberkorn & Panelist & University of Heidelberg & Germany \\
Ken Herrmann & Project leader & University Hospital of Essen & Germany \\
Michael Hofman & Panelist & Peter MacCallum Cancer Centre, Melbourne & USA \\
Thomas Hope & Panelist & University of California San Francisco & India \\
Rakesh Kumar & Panelist & All India Institute Of Medical Sciences, New Delhi \\
Daniela Oprea-Lager & Panelist & Amsterdam UMC, Vrije Universiteit Amsterdam & The Netherlands \\
Steven Rowe & Panelist & Johns Hopkins Baltimore & USA \\
Sarah Schwarzenboeck & Panelist & University of Rostock & Germany \\
\hline
\end{tabular}


Fig. 1 E-PSMA project timeline

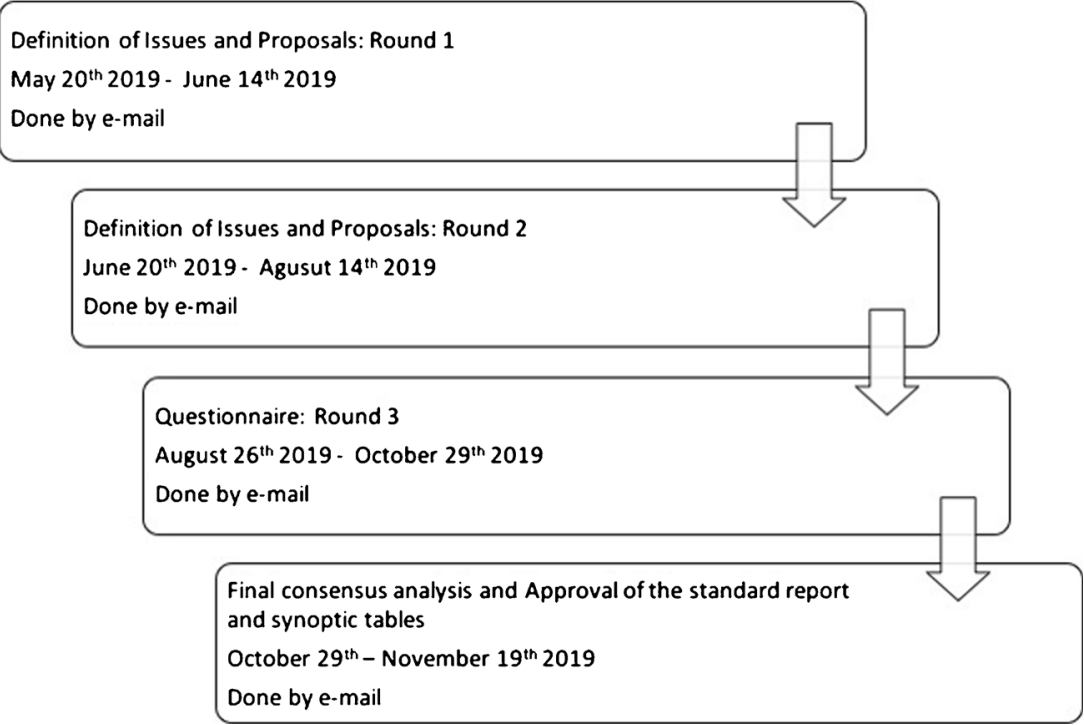

agreement; $0.21-0.40$ fair agreement; $0.41-0.60$ moderate agreement; 0.61-0.80 substantial agreement; 0.81-1.00 almost perfect agreement).
Fourteen out of sixteen questions reached an almost perfect concordance between panelists. Question nos. $13(k=0.61)$ and $14(k=0.55)$ reached only a moderate agreement between
Fig. 2 Open questions for panelists

\begin{tabular}{|c|c|c|c|}
\hline \# & Open Questions for Panelists & $\begin{array}{l}\text { Level of } \\
\text { Agreement }\end{array}$ & $\begin{array}{l}\text { Agreed } \\
\text { Answer }\end{array}$ \\
\hline 1 & $\begin{array}{l}\text { Do you think PSMA standardized and structured report is } \\
\text { necessary? }\end{array}$ & $\mathrm{k}=1.0$ & In favour \\
\hline 2 & $\begin{array}{l}\text { Do you agree with the inclusion of synoptic tables in the report } \\
\text { (Synoptic Table } 1 \text { and 2), in order to provide more reproducible } \\
\text { information? }\end{array}$ & $k=1.0$ & In favour \\
\hline 3 & $\begin{array}{l}\text { Do you agree with the inclusion of technical information in the } \\
\text { report (as described in Synoptic Table 1)? }\end{array}$ & $k=1.0$ & In favour \\
\hline 4 & $\begin{array}{l}\text { Do you think that reader experience (in reading PSMA images) } \\
\text { should be disclosed in the report? }\end{array}$ & $k=1.0$ & Against \\
\hline 5 & $\begin{array}{l}\text { Do you agree with the proposed } 5 \text {-point scale to rate the quality } \\
\text { of the scan as reported in Synoptic Table } 1 \text { ? (Quality scale: } 1= \\
\text { very poor/non-diagnostic; } 2=\text { poor; } 3=\text { moderate; } 4=\text { good; } \\
5=\text { excellent) }\end{array}$ & $\mathrm{k}=0.91$ & Against \\
\hline 6 & $\begin{array}{l}\text { Do you agree with the standard acquisition protocol (vertex to } \\
\text { mid-thigh)? }\end{array}$ & $k=1.0$ & In favour \\
\hline 7 & $\begin{array}{l}\text { Do you agree with the whole-body acquisition protocol (lower } \\
\text { extremities included) in those patients eligible for radio-ligand } \\
\text { therapy? }\end{array}$ & $\mathrm{k}=0.82$ & In favour \\
\hline 8 & $\begin{array}{l}\text { Do you agree with the use of miTNM (PROMISE criteria) for the } \\
\text { anatomical identification of the lesions (region-based)? }\end{array}$ & $k=1.0$ & In favour \\
\hline 9 & $\begin{array}{l}\text { Do you agree with the inclusion of visual PSMA expression (PSMA } \\
\text { Expression V - Synoptic Table 2) in the report? }\end{array}$ & $k=1.0$ & In favour \\
\hline 10 & $\begin{array}{l}\text { Do you agree with the proposed } 4 \text {-point scale ( } 0 \text { to } 3 \text { ) for PSMA } \\
\text { Expression V? }\end{array}$ & $\mathrm{k}=1.0$ & In favour \\
\hline 11 & $\begin{array}{l}\text { Do you agree with the inclusion of quantitative PSMA expression } \\
\text { (PSMA Expression Q - Synoptic Table 2) in the report? }\end{array}$ & $k=1.0$ & In favour \\
\hline 12 & $\begin{array}{l}\text { Do you agree with the use of tumor to background ratio (TBR) } \\
\text { instead of SUVmax for PSMA Expression Q? }\end{array}$ & $k=0.82$ & Against \\
\hline 13 & $\begin{array}{l}\text { Do you agree with the inclusion of PSMA-RADS in the report } \\
\text { (Synoptic Table 2), as method to evaluate reader confidence? }\end{array}$ & $\mathrm{k}=0.61$ & $\begin{array}{l}\text { Not } \\
\text { reached }\end{array}$ \\
\hline 14 & Do you agree with the proposed 5-point scale for PSMA-RADS? & $k=0.55$ & $\begin{array}{l}\text { Not } \\
\text { reached }\end{array}$ \\
\hline 15 & $\begin{array}{l}\text { Do you agree with the use of sub-categories (1A and } 1 B-3 A, 3 B \text {, } \\
3 C, 3 D) \text { for PSMA-RADS? }\end{array}$ & $k=0.73$ & Against \\
\hline 16 & $\begin{array}{l}\text { Do you agree with the inclusion of eventual incidental findings in } \\
\text { the report? }\end{array}$ & $k=1.0$ & In favour \\
\hline
\end{tabular}


experts. At question nos. 1, 2, 3, 6, 7, 8, 9, 10, 11, and 16, the agreement was in favor of the topic proposed. Therefore, structured report, synoptic tables, technical information, type of acquisition, staging criteria, visual and quantitative PSMA expression, and incidental findings have been included in EPSMA reporting guidelines.

At questions 4, 5, 12, and 15, the agreement was against the topic proposed. Therefore, reader experience, quality of the scan, TBR instead of $\mathrm{SUV}_{\max }$, and the use of sub-categories in the 5point scales were not included in E-PSMA reporting guidelines. Since questions 13 and 14 did not reach consensus, PSMARADS criteria [20] were not included in the final report. However, considering comments and proposal made in rounds 1 and 2, all panelists agreed in the inclusion of a modified 5-point scale to reflect the likelihood of the presence of PCa-related lesions.

\section{The EANM standardized reporting guidelines: E-PSMA-imaging methodology, structured report, and synoptic tables}

According to the findings obtained by modified Delphi consensus process, panelist recommendations were implemented in a structured report for PSMA-PET. Expert recommendations are reported below. PSMA-PET report template is reported in Appendix 1 (Supplemental Material). The synoptic tables are reported in Appendix 2 and 3 (Supplemental Material).

\section{Imaging methodology}

Many technical factors relating to methodology may affect the quality of a PET image acquisition. As these methodological aspects may influence the quality and interpretation of PSMAPET images, they should be described in each PSMA-PET report. The head of the report should include synoptic tables to summarize PSMA-PET technical data (synoptic Table 1).

Tracer activity used should be reported in $\mathrm{MBq}$, whether fixed (333 MBq for $\left.\left[{ }^{18} \mathrm{~F}\right] \mathrm{DCFPyL}\right)$ or patient-specific (1.8$2.2 \mathrm{MBq} / \mathrm{Kg}$ for $\left[{ }^{68} \mathrm{Ga}\right] \mathrm{Ga}-P S M A-11$ and $4 \mathrm{MBq} / \mathrm{Kg}$ for $\left[{ }^{18} \mathrm{~F}\right]$ PSMA-1007). In case a different tracer dosage is used, this should be reported.

As tumor PSMA uptake does not plateau within commonly used intervals, uptake intervals may affect lesion-tobackground contrast, detection rates, and quantitative reads. Therefore, the uptake interval between tracer injection and imaging should be reported. When used, type of diuretics and dosage should be reported.

The type of CT protocol (low dose vs. diagnostic) and use of oral or intravenous contrast should be reported. If intravenous contrast is used, consideration should be given to imaging in the urography phase to better delineate the ureters and bladder, in the staging or biochemical recurrence setting.
The standard acquisition should be from the vertex to the midthigh. In patients where there is known disease or concern for disease in the lower extremities, the acquisition can be extended (total-body acquisition). Total-body acquisition can be considered also in patients eligible for radioligand PSMA-based therapy.

The overall quality of the study should be assessed. This judgment is based on reader personal experience and it is aimed to evaluate the reproducibility of the scan. However, in consideration of experts' recommendation, this information should not be included in the report.

\section{The structured report}

An overview of all items necessary for reporting of findings on PSMA-PET that can be clinically used is provided in synoptic Table 2. Synoptic Table 2 should be included at the end of the clinical report as the final summary.

\section{Patient history}

Depending on the clinical indication for PSMA-PET, several variables in a patient's history will influence his a priori chance of having primary prostate cancer, local recurrence, lymph node metastases, or distant metastatic disease [55-58].

In newly diagnosed patients, clinical tumor stage, PSA level, and Gleason score influence the probability of having lymph node metastases $[59,60]$. Also at biochemical recurrence, the probability of scan positivity will depend on the patients' PSA level and its kinetics (namely PSA doubling time (PSAdt)), together with the clinical setting of PSA relapse (biochemical persistence vs. first biochemical recurrence vs. advanced metastatic disease) [55-58, 61, 62]. Therefore, knowledge of risk factors such as PSA level, PSAdt, Gleason score, clinical or pathological tumor stage, and prior and/or current treatment(s) is necessary to adequately report on findings on PSMA-PET.

ADT might modulate levels of PSMA expression over time, and its use and timing should be included in reports, especially for comparison of longitudinally repeated PSMAPET $[5,52]$.

Table 2 Qualitative evaluation of PSMA expression through a 4-point scale

PSMA expression Grade of PSMA expression $\mathrm{V}$ (visual score)

\begin{tabular}{ll}
\hline Score $=0$ & Below blood pool \\
Score $=1$ & Equal to or above blood pool and lower than liver \\
Score $=2$ & Equal to or above liver and lower than parotid gland \\
Score $=3$ & Equal to or above parotid gland \\
\hline
\end{tabular}


The clinical setting of recurrence (biochemical persistence vs. biochemical recurrence vs. advanced disease) as well as the scenario of hormone-sensitive PCa (HSPC) vs. CRPC should be included as well $[55,57]$.

\section{General consideration}

Describing PSMA uptake in either prostate, prostate bed, or metastases (lymph node, bone, or visceral soft tissue) should include both qualitative and quantitative descriptions, as reported in synoptic Table 2. Visual description (PSMA expression V) should relate PSMA uptake to background uptake in the blood, liver, and salivary glands on a visual scale $0-3$, as reported in Table 2. Quantitative description (PSMA Expression Q) should preferably include $\mathrm{SUV}_{\max }$ or, alternatively, a tumor-to-background ratio.

According to experts' recommendation (question 8), reports on primary tumor/prostate bed recurrence and metastases should include TNM classification (molecular imaging TM (miTNM)), as proposed by PROMISE criteria [19] and as reported in Table 3.

Suspected PCa site(s) (e.g., prostate/prostate bed, lymph node station, bone structure, organs), anatomical size, and number of lesions (oligo vs. multi-metastatic disease) should be reported.

Finally, the report should include a 5-point scale [20], as framework for classifying individual findings into categories that reflect the likelihood of the presence of $\mathrm{PCa}$, as reported

Table 3 Regional classification of PSMA-PET findings

\begin{tabular}{ll}
\hline Class & Description \\
\hline Local tumor (T) & \\
miT0 & No local tumor \\
miT2 & Organ-confined tumor \\
miT3a & Non-organ-confined tumor (extracapsular extension) \\
miT3b & Non-organ-confined tumor (seminal vesicles invasion) \\
miT4 & Tumor invading adjacent structures (other than \\
& seminal vesicles) \\
miTr & Presence of local recurrence after radical prostatectomy \\
Regional nodes & N) \\
miN0 & No positive regional lymph nodes \\
miN1 & Positive regional lymph nodes \\
Distant metastases (M) \\
miM0 & No distant metastases \\
miM1a & Extra-pelvic lymph nodes \\
miM1b & Bone metastasis \\
miM1c & Non-nodal visceral metastasis: report involved organ(s) \\
\hline
\end{tabular}

Adapted from Eiber M, et al. Prostate cancer molecular imaging standardized evaluation (PROMISE): proposed miTNM classification for the interpretation of PSMA-ligand PET/CT. J Nucl Med. 2018 Mar;59(3):469-478 in Table 4. The number of findings should be reported for each region, as defined by the miTNM criteria. In case of multiple metastases (e.g., more than five), as might happen in advanced patients, readers can add the definition of "multi-metastatic or poly-metastatic" in the specific region of dissemination. In case of poly-metastatic disease, data about lesion quantification should be calculated considering the five most evident metastases per region (to be selected based on size and intensity).

\section{Region-based analysis: prostate and prostate bed}

In the initial stage of $\mathrm{PCa}$, reporting on local tumor stage should include uni vs. multifocal disease, laterality (unilateral or bilateral), localization (apical, median, or basal), and (whenever possible) suspicion of extracapsular extension (ECE) or seminal vesical invasion (SVI). Reporting ECE and SVI should preferably include anatomical description from diagnostic CT (if available). Reporting on potential recurrence in prostate bed should include laterality and localization (anastomosis vs. posterior) and uni vs. multifocal disease.

\section{Region-based analysis: lymph nodes}

Lymph node size is not always directly related to chance of PCa metastases [63]. Still, the likelihood of PET positivity does depend on size, as microscopic metastases (e.g., $\leqslant 3 \mathrm{~mm}$ ) will likely remain below PET detection limits. This contributes to false-negative findings of PSMA-PET for lymph node staging in primary $\mathrm{PCa}[59,60,64]$. In the biochemical recurrence setting, as histopathological confirmation is often lacking, caution for false positives is mandated [33, 39]. Reporting should include the exact station of lymph node metastases and the short-axis measurement. Also, pelvic lymph node descriptions should include where nodes are located, to help urologist to interpret whether PSMA-PET-positive lymph nodes are within or outside surgical dissection templates. Whenever possible, chance of malignancy should be related to quantitative measurements and reader's confidence (Table 4).

\section{Region-based analysis: skeleton}

One of the pitfalls of PSMA-PET is the rate of false-positive bone lesions. The exact cause of false positivity is not known, but may be attributed to bone degeneration, traumatic injury, or benign bone lesions. It should be noted that false positivity rate for bone lesions may differ between PSMA ligands [41]. Solitary bone lesions detected in patients with no metastases otherwise should be interpreted with caution, especially in pre-surgery setting. Reports should include clinical characteristics (e.g., pain, PSA kinetics), and correlation with other modalities including MRI, contrast-enhanced CT, and bone scan. When there is uncertainty and histopathological 
Table 4 Interpretation of PSMAPET findings according to the reader confidence expressed through a 5-point scale

\begin{tabular}{ll} 
Score & Definition \\
\hline 1 & $\begin{array}{l}\text { Benign lesion without abnormal PSMA uptake } \\
2\end{array}$ \\
$\begin{array}{c}\text { Probably benign lesion: faint PSMA uptake (equal or lower than background) in a site atypical for } \\
\text { prostate cancer }\end{array}$ \\
$\begin{array}{c}\text { Equivocal finding: faint uptake in a site typical for prostate cancer or intense uptake in a site atypical for } \\
\text { prostate cancer }\end{array}$ \\
$\begin{array}{c}\text { Probably prostate cancer: intense uptake in typical site of prostate cancer, but without definitive findings } \\
\text { on CT* }\end{array}$ \\
$\begin{array}{c}\text { Definitive evidence of prostate cancer: intense uptake in typical site of prostate cancer, with definitive } \\
\text { findings on CT }\end{array}$
\end{tabular}

Adapted from Werner RA, et al. Recent updates on molecular imaging reporting and data systems (MI-RADS) for theranostic radiotracers-navigating pitfalls of SSTR- and PSMA-targeted PET/CT. J Clin Med. 2019 Jul 19;8(7)

*A definitive finding on CT means the presence of a real anatomical substrate on the CT confirmation is feasible, it should be recommended to referring physicians in reports.

\section{Region-based analysis: non-nodal visceral soft tissue}

Detection or suspicion of visceral metastases should include reporting of localization and PSMA expression levels in relation to background uptake qualitatively (PSMA expression V, Table 2) and quantitatively (PSMA expression Q). Presence of visceral metastases should be reported with anatomical information from CT and related to clinical characteristics (e.g., PSA levels and comorbidities), to prevent detection of false positives [33].

\section{E-PSMA reporting system in staging, recurrent setting, advanced setting, and response to therapy: a clinical summary}

The use of PSMA-PET is increasing in routine clinical practice both in initial staging of PCa and for the localization of biochemically recurrent $\mathrm{PCa}$. The accurate detection of malignant PCa lesions has a major impact on management decisions and may result in withholding definitive local therapy or lead to metastasis-directed therapy [65].

\section{Primary staging}

In primary staging, early detection of metastases is essential. Patients with proven metastatic disease are usually treated differently than patients with localized PCa. The detection of any additional lesion may change patient management and result in local radiotherapy, extended lymph node dissection, (oligo)metastases-directed therapy, or systemic (palliative) treatment. In a systematic review [66], high variation in sensitivity (33-92\% on a per-lesion analysis) with overall optimal specificity (82-100\% on a per-lesion analysis) was found in the detection of lymph node metastases, correlated by histopathological evaluation (extended pelvic lymph node dissection). Additionally, the primary tumor is nearly always detected by PSMA-PET, and PET metrics correlated with histologic grades (ISUP classification) [67, 68]. Analyses regarding the diagnostic accuracy of ${ }^{18} \mathrm{~F}$-labeled PSMA-PET in primary staging are ongoing. In yet unpublished results of a prospective trial, a sensitivity of $30.6-41.9 \%$ of $\left[{ }^{18} \mathrm{~F}\right] \mathrm{DCFPyL}$ PET for the detection of lymph node metastases was determined [68]. Regarding [ $\left.{ }^{18} \mathrm{~F}\right] 1007-P S M A-P E T$, local staging appears a promising technique, considering the low urinary excretion of this radiotracer [69].

Recently, in an Australian, multi-center, randomized, phase III clinical trial (proPSMA) [70], $\left[{ }^{68} \mathrm{Ga}\right] \mathrm{Ga}$-PSMA-11 PET provided greater accuracy in identifying nodal and distant metastases vs. conventional imaging (CT and bone scan) prior to curative-intent surgery or radiotherapy in high-risk PCa. Furthermore, $\left[{ }^{68} \mathrm{Ga}\right] \mathrm{Ga}-\mathrm{PSMA}-11$ PET vs. conventional imaging was associated with change in management in $28 \%$ vs. $15 \%$ of patients and was associated with a lower percentage of equivocal findings (7\% vs. $23 \%$ ). Finally, even if both imaging techniques involve exposure to radiation, the dose associated with $\left[{ }^{68} \mathrm{Ga}\right] \mathrm{Ga}-\mathrm{PSMA}-11$ was less than half that associated with conventional imaging ( $8.4 \mathrm{mSv}$ vs. $19.2 \mathrm{mSv})$.

Regarding these considerations, PSMA-PET is a suitable replacement for conventional imaging, providing superior accuracy, to the combined findings of CT and bone scanning, in patients with high risk of nodal involvement [71], while patients at lower risk should be spared by this imaging procedure.

\section{Recurrent setting}

The detection rate of metastases (i.e., percentage positive scans) of PSMA-PET in patients with BCR has been studied intensively. A recent meta-analysis [11] showed an overall detection rate in patients with BCR of $76 \%$. At low PSA values $(<0.5 \mathrm{ng} /$ $\mathrm{mL}$ ), detection of metastases was $45 \%$. Furthermore, a recently published large prospective study showed comparable results 
[14]. The positive predictive value (PPV) of $\left[{ }^{68} \mathrm{Ga}\right] \mathrm{Ga}$-PSMA11 PET has been calculated as $92 \%$ [14]. For ${ }^{18} \mathrm{~F}$-labeled PSMA (namely $\left[{ }^{18} \mathrm{~F}\right] \mathrm{DCFPyL}$ and $\left[{ }^{18} \mathrm{~F}\right]$ PSMA-1007), fewer results concerning the diagnostic accuracy in patients with $\mathrm{BCR}$ are available at present. For $\left[{ }^{18} \mathrm{~F}\right] \mathrm{DCFPyL}$, detection rates ranging from 84.6 to $86.3 \%$ have been documented, with a detection at low PSA values $(<0.5 \mathrm{ng} / \mathrm{mL})$ of $60 \%[62,72]$. Preliminary results of a large prospective multicenter trial [73] showed a PPV of $84.5 \%$ for $\left[{ }^{18}\right.$ F]DCFPyL PET. Similar results have been described for $\left[{ }^{18} \mathrm{~F}\right] \mathrm{PSMA}-1007[74,75]$.

At present, EAU guidelines suggest performing PSMAPET in any case of proven BCR [8]. However, the incidence of false-negative scans is not negligible. In recurrent setting, PSMA-PET detection rate is influenced by several factors as recently reported in some prediction models $[55,56,58,76]$. PSA, as expression of tumor burden, is not the only influencing parameter. PSAdt and Gleason score as expression of tumor aggressiveness together with the administration of concurrent ADT are parameters able to influence the likelihood of a positive scan [76]. While reporting PSMA-PET in recurrent setting, also the clinical stage of the disease should be taken into consideration $[55,57]$. Persistent disease after surgery (detectable PSA levels after surgery) [61] and BCR (undetectable PSA levels after surgery), while both represent an early recurrence, are two conditions with different outcome and different incidence of detectable metastatic disease [55, 57, 61]. Finally, the proper knowledge of potential pitfalls during PET image interpretation, while probably reducing PSMAPET sensitivity, will increase its overall specificity [33].

\section{Advanced setting and assessment of the response to systematic therapy}

The role of PSMA-PET in advanced setting presents less level of evidence compared to initial staging and BCR. Nonmetastatic CRPC (nmCRPC) is a condition characterized by a rising PSA level, castrate testosterone levels, and no evidence of distant metastases by conventional bone scan and cross-sectional imaging of the chest, abdomen, and pelvis [8]. This clinical scenario became recently of high interest, since new androgen receptor-targeted therapies have been recently approved in this stage (SPARTAN, PROSPER, and ARAMIS trials). In the setting, PSMA-PET proved its ability to detect $\mathrm{PCa}$ locations in patients negative at conventional imaging (nmCRPC). Thus, as recently stated by the EAU consensus panel in advanced PCa [16], ASCO guidelines [15], and Advanced PCa Consensus Conference (APCC) [9], it should also be recognized that the majority of patients in clinical trials who benefited from the addition of nextgeneration ADT would probably have had positive PSMAPET imaging results. It is uncertain whether stratification based on PSMA-PET would identify subgroups of patients (e.g., those with distant rather than local or loco-regional disease) that benefit most. Tumor heterogeneity is a key event in advanced PCa. Tumor cells exhibit different phenotypes and, accordingly, PSMA might not be over-expressed in all metastatic sites. This event should be taken into consideration while reporting PET scan in $\mathrm{mCRPC}$, namely while evaluating the response to systemic therapy.

Regarding the response to therapy assessment, the Prostate Cancer Clinical Trials Working Group (PCWG) criteria include clinical and laboratory parameters, as well as conventional imaging modalities such as CT and bone scan findings but advanced molecular imaging techniques are not yet considered. PSMAPET is not yet validated for response assessment, especially in the context of clinical trials [77]. Recently, consensus statement criteria for response evaluation using PSMA-PET were developed [16]. The statements regarded both the utility and the best time to perform PSMA-PET, as well as the optimal strategy to select patients who may benefit from treatments and criteria to be used for evaluation of response when using different types of PSMA tracers. Consensus was met on the utility of PSMAPET for response assessment in patients with metastatic PCa, irrespective of the moment and type of treatment used (i.e., local or systemic), but solely in cases when clinical management is expected and with a 3-month interval after initiation of therapy in HSPC. Proposed criteria should only be adopted in the context of clinical trials, preferably by dividing patients in responders and non-responders. The category of responders is including the whole spectrum of patients presenting with stable disease, partial and complete response, while the non-responders are patients with progressive disease on PSMA-PET imaging. The prerequisite of robust and reproducible interpretation of response to treatment when using PSMA-PET scans is adequate semiquantitative evaluation. Consensus was reached on the use of SUV parameters for this purpose, by optimizing and harmonizing the protocols. Tools to estimate the total tumor burden represent a feasible alternative to reduce interobserver variability, being currently developed. However, one major issue is how to best define disease progression. Recently, PSMA-PET Progression (PPP) criteria have been proposed [78]. PPP defines PSMA treatment response in three different criteria: (1) appearance of 2 or more new PSMA-positive distant lesions, (2) appearance of 1 new PSMA-positive lesion plus consistent clinical and/or laboratory data and recommended confirmation by biopsy or correlative imaging within 3 months of PSMA-PET, and (3) increase in size or PSMA uptake of 1 or more existing lesions by $30 \%$ plus consistent clinical and/or laboratory data and/or confirmation by biopsy or correlative imaging within 3 months of PSMA-PET. These criteria should be taken into consideration while reporting PSMA-PET in patients undergoing systemic therapies.

Finally, the growing interest in PSMA-targeted therapies is not unnoticed. One phase 3 trial (VISION, NCT03511664) and one phase 2 trial (TheraP, NCT03392428) [79] are currently ongoing. Both trials use PSMA-PET to identify patients with high PSMA expression, who are suitable candidates for 
$\left[{ }^{177} \mathrm{Lu}\right] \mathrm{Lu}-\mathrm{PSMA}-617$ therapy, but they use different PET imaging thresholds to define suitability. In addition, the TheraP trial uses $2-\left[{ }^{18} \mathrm{~F}\right]$ FDG PET to assist in identifying sites of PSMA-negative disease that cannot be targeted with $\left[{ }^{177} \mathrm{Lu}\right]$ Lu-PSMA-617. These patients have been shown to have a poor prognosis [80]. Finally, studies indicate that both intrapatient and interpatient PSMA expressions are highly heterogeneous in patients candidate for PSMA-targeted therapy, and that many of them express little or no PSMA. This consideration should be clearly stated and defined while interpreting PSMA-PET in advanced setting [81, 82].

Supplementary Information The online version contains supplementary material available at https://doi.org/10.1007/s00259-021-05245-y.

Acknowledgements The authors would like to thank the European Association of Nuclear Medicine (EANM) and the EANM Oncological and Theranostic Committee for supporting this project.

Author contribution Design of the project: FC, SF, and KH. Methodology: FC, DOL, LE, SF, and KH. Questionnaires: all authors. Delphi rounds: all authors. Manuscript Drafting: FC, DOL, LE, and JA. Manuscript review: all authors. Project supervision: FC, SF, and KH. All authors read and approved the final manuscript.

Funding Open Access funding provided by Amsterdam UMC (Vrije Universiteit Amsterdam).

\section{Declarations}

Ethics approval All procedures performed in studies involving human participants were in accordance with the ethical standards of the institutional and/or national research committee and with the principles of the 1964 Declaration of Helsinki and its later amendments or comparable ethical standards.

Conflict of interest The authors declare no competing interests.

Open Access This article is licensed under a Creative Commons Attribution 4.0 International License, which permits use, sharing, adaptation, distribution and reproduction in any medium or format, as long as you give appropriate credit to the original author(s) and the source, provide a link to the Creative Commons licence, and indicate if changes were made. The images or other third party material in this article are included in the article's Creative Commons licence, unless indicated otherwise in a credit line to the material. If material is not included in the article's Creative Commons licence and your intended use is not permitted by statutory regulation or exceeds the permitted use, you will need to obtain permission directly from the copyright holder. To view a copy of this licence, visit http://creativecommons.org/licenses/by/4.0/.

\section{References}

1. O'Keefe DS, Bacich DJ, Huang SS, Heston WDW. A perspective on the evolving story of PSMA biology, PSMA-based imaging, and endoradiotherapeutic strategies. J Nucl Med. 2018;59(7):1007-13.
2. Gordon IO, Tretiakova MS, Noffsinger AE, Hart J, Reuter VE, AlAhmadie HA. Prostate-specific membrane antigen expression in regeneration and repair. Mod Pathol. 2008;21(12):1421-7.

3. Kaittanis C, Andreou C, Hieronymus H, et al. Prostate-specific membrane antigen cleavage of vitamin B9 stimulates oncogenic signaling through metabotropic glutamate receptors. J Exp Med. 2018;215(1):159-75. https://doi.org/10.1084/jem.20171052.

4. Aggarwal R, Wei X, Kim W, et al. Heterogeneous flare in prostatespecific membrane antigen positron emission tomography tracer uptake with initiation of androgen pathway blockade in metastatic prostate cancer. Eur Urol Oncol. 2018;1(1):78-82.

5. Emmett L, Yin C, Crumbaker M, et al. Rapid modulation of PSMA expression by androgen deprivation: serial 68Ga-PSMA-11 PET in men with hormone-sensitive and castrate-resistant prostate cancer commencing androgen blockade. J Nucl Med. 2019;60(7):950-4.

6. Fendler WP, Weber M, Iravani A, et al. Prostate-specific membrane antigen ligand positron emission tomography in men with nonmetastatic castration-resistant prostate cancer. Clin Cancer Res. 2019;25(24):7448-54.

7. Eder M, Schäfer M, Bauder-Wüst U, Hull W-E, Wängler C, Mier $\mathrm{W}$, et al. 68Ga-complex lipophilicity and the targeting property of a urea-based PSMA inhibitor for PET imaging. Bioconjug Chem. 2012;23(4):688-97.

8. Mottet N, Cornford P, van den Bergh RCN, Briers E, De Santis M, Fanti S, et al. EAU-ESTRO-SIOG guidelines on prostate cancer. EAU Guidelines. Edn. presented at the EAU Annual Congress Amsterdam 2020. ISBN 978-94-92671-07-3.

9. Gillessen S, Omlin A, Attard G, et al. Management of patients with advanced prostate cancer: recommendations of the St Gallen Advanced Prostate Cancer Consensus Conference (APCCC) 2015. Ann Oncol. 2019;30(12):e3.

10. Gillessen S, Attard G, Beer TM, et al. Management of patients with advanced prostate cancer: report of the Advanced Prostate Cancer Consensus Conference 2019 [published online ahead of print, 2020 Jan 27]. Eur Urol. 2020:S0302-2838(20)30048-8.

11. Perera M, Papa N, Roberts M, et al. Gallium-68 prostate-specific membrane antigen positron emission tomography in advanced prostate cancer-updated diagnostic utility, sensitivity, specificity, and distribution of prostate-specific membrane antigen-avid lesions: a systematic review and meta-analysis. Eur Urol. 2020;77(4):403-17.

12. Calais J, Ceci F, Eiber M, et al. 18F-fluciclovine PET-CT and 68Ga-PSMA-11 PET-CT in patients with early biochemical recurrence after prostatectomy: a prospective, single-centre, single-arm, comparative imaging trial [published correction appears in Lancet Oncol. 2019 Nov;20(11):e613] [published correction appears in Lancet Oncol. 2020 Jun;21(6):e304]. Lancet Oncol. 2019;20(9): 1286-94.

13. Morigi JJ, Stricker PD, van Leeuwen PJ, et al. Prospective comparison of $18 \mathrm{~F}$-fluoromethylcholine versus $68 \mathrm{Ga}$-PSMA PET/CT in prostate cancer patients who have rising PSA after curative treatment and are being considered for targeted therapy. J Nucl Med. 2015;56(8):1185-90. https://doi.org/10.2967/jnumed.115.160382.

14. Fendler WP, Calais J, Eiber M, et al. Assessment of 68Ga-PSMA11 PET accuracy in localizing recurrent prostate cancer: a prospective single-arm clinical trial. JAMA Oncol. 2019;5(6):856-63.

15. Trabulsi EJ, Rumble RB, Jadvar H, et al. Optimum imaging strategies for advanced prostate cancer: ASCO Guideline [published online ahead of print, 2020 Jan 15]. J Clin Oncol. 2020: JCO1902757.

16. Fanti S, Goffin K, Hadaschik BA, et al. Consensus statements on PSMA PET/CT response assessment criteria in prostate cancer [published online ahead of print, $2020 \mathrm{Jul}$ 2]. Eur J Nucl Med Mol Imaging. 2020. https://doi.org/10.1007/s00259-020-04934-4.

17. Kratochwil C, Fendler WP, Eiber M, et al. EANM procedure guidelines for radionuclide therapy with 177Lu-labelled PSMA-ligands 
(177Lu-PSMA-RLT). Eur J Nucl Med Mol Imaging. 2019;46(12): 2536-44. https://doi.org/10.1007/s00259-019-04485-3.

18. Fanti S, Minozzi S, Morigi JJ, Giesel F, Ceci F, Uprimny C, et al. Development of standardized image interpretation for 68Ga-PSMA PET/CT to detect prostate cancer recurrent lesions. Eur J Nucl Med Mol Imaging. 2017;44(10):1622-35.

19. Eiber M, Herrmann K, Calais J, Hadaschik B, Giesel FL, Hartenbach M, et al. Prostate cancer molecular imaging standardized evaluation (PROMISE): proposed miTNM classification for the interpretation of PSMA-ligand PET/CT. J Nucl Med. 2018;59(3):469-78.

20. Werner RA, Thackeray JT, Pomper MG, Bengel FM, Gorin MA, Derlin $\mathrm{T}$, et al. Recent updates on molecular imaging reporting and data systems (MI-RADS) for theranostic radiotracers-navigating pitfalls of SSTR- and PSMA-targeted PET/CT. J Clin Med. 2019;8(7):pii: E1060.

21. Toriihara A, Nobashi T, Baratto L, et al. Comparison of three interpretation criteria of 68Ga-PSMA11 PET based on inter- and intrareader agreement [published online ahead of print, 2019 Sep 27]. J Nucl Med. 2019:jnumed.119.232504. https://doi.org/10.2967/ jnumed.119.232504.

22. Fendler WP, Eiber M, Beheshti M, Bomanji J, Ceci F, Cho S, et al. (68)Ga-PSMA PET/CT: joint EANM and SNMMI procedure guideline for prostate cancer imaging: version 1.0. Eur J Nucl Med Mol Imaging. 2017;44(6):1014-24.

23. Chen Y, Pullambhatla M, Foss CA, Byun Y, Nimmagadda S, Senthamizhchelvan S, et al. 2-(3-\{1-Carboxy-5-[(6-[18F]fluoropyridine-3-carbonyl)-amino]-pentyl\}-ureido)-pen tanedioic acid, [18F]DCFPyL, a PSMA-based PET imaging agent for prostate cancer. Clin Cancer Res. 2011;17(24):7645-53.

24. Mease RC, Dusich CL, Foss CA, et al. N-[N-[(S)-1,3Dicarboxypropyl]carbamoyl]-4-[18F]fluorobenzyl-L-cysteine, [18F]DCFBC: a new imaging probe for prostate cancer. Clin Cancer Res. 2008;14(10):3036-43. https://doi.org/10.1158/10780432.CCR-07-1517.

25. Sanchez-Crespo A. Comparison of Gallium-68 and Fluorine-18 imaging characteristics in positron emission tomography. Appl Radiat Isot. 2013;76:55-62.

26. Wondergem M, Jansen BHE, van der Zant FM, et al. Early lesion detection with $18 \mathrm{~F}-\mathrm{DCFPyL}$ PET/CT in 248 patients with biochemically recurrent prostate cancer. Eur J Nucl Med Mol Imaging. 2019;46(9):1911-8.

27. Giesel FL, Knorr K, Spohn F, et al. Detection efficacy of 18FPSMA-1007 PET/CT in 251 patients with biochemical recurrence of prostate cancer after radical prostatectomy. J Nucl Med. 2019;60(3):362-8.

28. Zlatopolskiy BD, Endepols H, Krapf P, Guliyev M, Urusova EA, Richarz R, et al. Discovery of (18)F-JK-PSMA-7, a PET probe for the detection of small PSMA-positive lesions. J Nucl Med. 2019;60(6):817-23.

29. Eiber M, Kroenke M, Wurzer A, et al. 18F-rhPSMA-7 PET for the detection of biochemical recurrence of prostate cancer after radical prostatectomy. J Nucl Med. 2020;61(5):696-701.

30. Pfob CH, Ziegler S, Graner FP, Köhner M, Schachoff S, Blechert $\mathrm{B}$, et al. Biodistribution and radiation dosimetry of 68Ga-PSMA HBED CC - a PSMA specific probe for PET imaging of prostate cancer. Eur J Nucl Med Mol Imaging. 2016;43(11):1962-70.

31. Giesel FL, Hadaschik B, Cardinale J, Radtke J, Vinsensia M, Lehnert W, et al. F-18 labelled PSMA-1007: biodistribution, radiation dosimetry and histopathological validation of tumor lesions in prostate cancer patients. Eur J Nucl Med Mol Imaging. 2017;44(4): 678-88.

32. Pollard J, Raman C, Zakharia Y, et al. Quantitative test-retest measurement of 68Ga-PSMA-HBED-CC (PSMA-11) in tumor and normal tissue [published online ahead of print, 2019 Dec 5]. J
Nucl Med. 2019:jnumed.119.236083. https://doi.org/10.2967/ jnumed.119.236083.

33. Sheikhbahaei S, Afshar-Oromieh A, Eiber M, Solnes LB, Javadi MS, Ross AE, et al. Pearls and pitfalls in clinical interpretation of prostate-specific membrane antigen (PSMA)-targeted PET imaging. Eur J Nucl Med Mol Imaging. 2017;44(12):2117-36.

34. Hope TA, Goodman JZ, Allen IE, Calais J, Fendler WP, Carroll PR. Metaanalysis of 68Ga-PSMA-11 PET accuracy for the detection of prostate cancer validated by histopathology. J Nucl Med. 2019;60(6):786-93.

35. Ristau BT, O'Keefe DS, Bacich DJ. The prostate-specific membrane antigen: lessons and current clinical implications from 20 years of research. Urol Oncol. 2014;32(3):272-9.

36. Siva S, Callahan J, Pryor D, Martin J, Lawrentschuk N, Hofman MS. Utility of $68 \mathrm{Ga}$ prostate specific membrane antigen-positron emission tomography in diagnosis and response assessment of recurrent renal cell carcinoma. J Med Imaging Radiat Oncol. 2017:61(3):372-8.

37. Kesler M, Levine C, Hershkovitz D, et al. 68Ga-PSMA is a novel PET-CT tracer for imaging of hepatocellular carcinoma: a prospective pilot study [published online ahead of print, 2018 Jul 12]. J Nucl Med. 2018:jnumed.118.214833.

38. Sathekge M, Modiselle M, Vorster M, et al. ${ }^{68}$ Ga-PSMA imaging of metastatic breast cancer. Eur J Nucl Med Mol Imaging. 2015;42(9):1482-3.

39. Rischpler C, Beck TI, Okamoto S, et al. 68Ga-PSMA-HBED-CC uptake in cervical, celiac, and sacral ganglia as an important pitfall in prostate cancer PET imaging. J Nucl Med. 2018;59(9):1406-11.

40. Panagiotidis E, Paschali A, Giannoula E, Chatzipavlidou V. Rib fractures mimicking bone metastases in 18F-PSMA-1007 PET/ CT for prostate cancer. Clin Nucl Med. 2019;44(1):e46-e8.

41. Rauscher I, Krönke M, König M, et al. Matched-pair comparison of 68Ga-PSMA-11 PET/CT and 18F-PSMA-1007 PET/CT: frequency of pitfalls and detection efficacy in biochemical recurrence after radical prostatectomy. J Nucl Med. 2020;61(1):51-7.

42. Sasikumar A, Joy A, Nanabala R, Pillai MR, T AH. 68Ga-PSMA $\mathrm{PET} / \mathrm{CT}$ false-positive tracer uptake in Paget disease. Clin Nucl Med 2016;41(10):e454-e455.

43. Ribeiro AMB, Lima ENP, Rocha MM. Fibrous dysplasia as a possible false-positive finding in $68 \mathrm{Ga}$-labeled prostate-specific membrane antigen positron emission tomography/computed tomography study in the follow-up of prostate cancer. World J Nucl Med. 2019;18(4):409-12.

44. Noto B, Vrachimis A, Schafers M, Stegger L, Rahbar K. Subacute stroke mimicking cerebral metastasis in 68Ga-PSMA-HBED-CC PET/CT. Clin Nucl Med. 2016;41(10):e449-51.

45. Bilgin R, Ergul N, Cermik TF. Incidental meningioma mimicking metastasis of prostate adenocarcinoma in 68Ga-labeled PSMA ligand PET/CT. Clin Nucl Med. 2016;41(12):956-8.

46. Bertagna F, Albano D, Cerudelli E, Gazzilli M, Giubbini R, Treglia G. Potential of radiolabelled PSMA PET/CT or PET/MRI diagnostic procedures in gliomas/glioblastomas [published online ahead of print, 2019 Oct 16]. Curr Radiopharm. 2019. https://doi.org/10. 2174/1874471012666191017093721.

47. Hermann RM, Djannatian M, Czech N, Nitsche M. Prostatespecific membrane antigen PET/CT: false-positive results due to sarcoidosis? Case Rep Oncol. 2016;9(2):457-63.

48. Prasad V, Steffen IG, Diederichs G, Makowski MR, Wust P, Brenner W. Biodistribution of [68Ga]PSMA-HBED-CC in patients with prostate cancer: characterization of uptake in normal organs and tumour lesions. Mol Imaging Biol. 2016;18(3):428-36.

49. Bouchelouche K, Vendelbo MH. Pulmonary opacities and bronchiectasis avid on 68Ga-PSMA PET. Clin Nucl Med. 2017;42(4): e216-e7.

50. McGuiness M, Sounness B. 68Ga-PSMA-ligand PET/CT uptake in anthracosilicosis. Clin Nucl Med. 2017;42(10):e431-e2. 
51. Ahuja A, Taneja S, Thorat K, Jena A. 68Ga-prostate-specific membrane antigen-avid tubercular lesions mimicking prostate cancer metastasis on simultaneous prostate-specific membrane antigen PET/MRI. Clin Nucl Med. 2017;42(12):e509-e10.

52. Lückerath K, Wei L, Fendler WP, et al. Preclinical evaluation of PSMA expression in response to androgen receptor blockade for theranostics in prostate cancer. EJNMMI Res. 2018;8(1):96.

53. Sasikumar A, Joy A, Nair BP, Pillai MRA, Madhavan J. False positive uptake in bilateral gynecomastia on 68Ga-PSMA PET/ CT scan. Clin Nucl Med. 2017;42(9):e412-e4.

54. Tosoian JJ, Gorin MA, Rowe SP, Andreas D, Szabo Z, Pienta KJ, et al. Correlation of PSMA-targeted (18)F-DCFPyL PET/CT findings with Immunohistochemical and genomic data in a patient with metastatic neuroendocrine prostate cancer. Clin Genitourin Cancer. 2017;15(1):e65-e8.

55. Ceci F, Bianchi L, Borghesi M, et al. Prediction nomogram for 68Ga-PSMA-11 PET/CT in different clinical settings of PSA failure after radical treatment for prostate cancer. Eur J Nucl Med Mol Imaging. 2020;47(1):136-46.

56. Bianchi L, Borghesi M, Schiavina R, et al. Predictive accuracy and clinical benefit of a nomogram aimed to predict 68Ga-PSMA PET/ $\mathrm{CT}$ positivity in patients with prostate cancer recurrence and PSA $<$ $1 \mathrm{ng} / \mathrm{ml}$ external validation on a single institution database [published online ahead of print, 2020 Jan 31]. Eur J Nucl Med Mol Imaging. 2020. https://doi.org/10.1007/s00259-020-04696-z.

57. Ceci F, Castellucci P, Graziani T, et al. 68Ga-PSMA-11 PET/CT in recurrent prostate cancer: efficacy in different clinical stages of PSA failure after radical therapy. Eur J Nucl Med Mol Imaging. 2019;46(1):31-9.

58. Rauscher I, Düwel C, Haller B, et al. Efficacy, predictive factors, and prediction nomograms for $68 \mathrm{Ga}$-labeled prostate-specific membrane antigen-ligand positron-emission tomography/ computed tomography in early biochemical recurrent prostate cancer after radical prostatectomy. Eur Urol. 2018;73(5):656-61.

59. Budaus L, Leyh-Bannurah SR, Salomon G, Michl U, Heinzer H, Huland $\mathrm{H}$, et al. Initial experience of (68)Ga-PSMA PET/CT imaging in high-risk prostate cancer patients prior to radical prostatectomy. Eur Urol. 2016;69(3):393-6.

60. Maurer T, Gschwend JE, Rauscher I, Souvatzoglou M, Haller B, Weirich G, et al. Diagnostic efficacy of (68)gallium-PSMA positron emission tomography compared to conventional imaging for lymph node staging of 130 consecutive patients with intermediate to high risk prostate cancer. J Urol. 2016;195(5):1436-43.

61. Farolfi A, Gafita A, Calais J, et al. 68Ga-PSMA-11 positron emission tomography detects residual prostate cancer after prostatectomy in a multicenter retrospective study. J Urol. 2019;202(6):117481.

62. Wondergem M, Jansen BHE, van der Zant FM, van der Sluis TM, Knol RJJ, van Kalmthout LWM, et al. Early lesion detection with (18)F-DCFPyL PET/CT in 248 patients with biochemically recurrent prostate cancer. Eur J Nucl Med Mol Imaging. 2019;46(9): 1911-8.

63. Tiguert R, Gheiler EL, Tefilli MV, Oskanian P, Banerjee M, Grignon DJ, et al. Lymph node size does not correlate with the presence of prostate cancer metastasis. Urology. 1999;53(2):367-71.

64. van Leeuwen PJ, Donswijk M, Nandurkar R, Stricker P, Ho B, Heijmink S, et al. Gallium-68-prostate-specific membrane antigen ((68) Ga-PSMA) positron emission tomography (PET)/computed tomography $(\mathrm{CT})$ predicts complete biochemical response from radical prostatectomy and lymph node dissection in intermediateand high-risk prostate cancer. BJU Int. 2019;124(1):62-8.

65. Han S, Woo S, Kim YJ, Suh CH. Impact of (68)Ga-PSMA PET on the management of patients with prostate cancer: a systematic review and meta-analysis. Eur Urol. 2018;74(2):179-90.
66. Corfield J, Perera M, Bolton D, Lawrentschuk N. (68)Ga-prostate specific membrane antigen (PSMA) positron emission tomography (PET) for primary staging of high-risk prostate cancer: a systematic review. World J Urol. 2018;36(4):519-27.

67. Eiber M, Weirich G, Holzapfel K, Souvatzoglou M, Haller B, Rauscher I, et al. Simultaneous (68)Ga-PSMA HBED-CC PET/ MRI improves the localization of primary prostate cancer. Eur Urol. 2016;70(5):829-36.

68. Rowe S, Gorin S, Pienta K, Siegel B, Carroll P, Pouliot F, et al. Results from the OSPREY trial: A prospective phase $2 / 3$ multicenter study of 18F-DCFPyL PET/CT imaging in patients with prostate cancer-examination of diagnostic accuracy. J Nucl Med. 2019;60:586.

69. Kesch C, Vinsensia M, Radtke JP, Schlemmer HP, Heller M, Ellert E, et al. Intraindividual comparison of (18)F-PSMA-1007 PET/CT, multiparametric MRI, and radical prostatectomy specimens in patients with primary prostate cancer: a retrospective, Proof-ofConcept Study. J Nucl Med. 2017;58(11):1805-10.

70. Hofman MS, Lawrentschuk N, Francis RJ, et al. Prostate-specific membrane antigen PET-CT in patients with high-risk prostate cancer before curative-intent surgery or radiotherapy (proPSMA): a prospective, randomised, multicentre study. Lancet. 2020;395(10231):1208-16.

71. Gandaglia G, Ploussard G, Valerio M, et al. A novel nomogram to identify candidates for extended pelvic lymph node dissection among patients with clinically localized prostate cancer diagnosed with magnetic resonance imaging-targeted and systematic biopsies. Eur Urol. 2019;75(3):506-14.

72. Rousseau E, Wilson D, Lacroix-Poisson F, et al. A prospective study on 18F-DCFPyL PSMA PET/CT imaging in biochemical recurrence of prostate cancer. J Nucl Med. 2019;60(11):1587-93.

73. Morris MJ, Carroll P, Probst S, Pouliot F, Saperstein L, Siegel BA. A prospective phase $2 / 3$ multicenter study of F-18-DCFPyL PET/ $\mathrm{CT}$ imaging in patients with prostate cancer: examination of diagnostic accuracy (OSPREY). J Clin Oncol. 2018;36(15).

74. Giesel FL, Knorr K, Spohn F, Will L, Maurer T, Flechsig P, et al. Detection efficacy of (18)F-PSMA-1007 PET/CT in 251 patients with biochemical recurrence of prostate cancer after radical prostatectomy. J Nucl Med. 2019;60(3):362-8.

75. Rahbar K, Afshar-Oromieh A, Seifert R, Wagner S, Schafers M, Bogemann M, et al. Diagnostic performance of (18)F-PSMA-1007 $\mathrm{PET} / \mathrm{CT}$ in patients with biochemical recurrent prostate cancer. Eur J Nucl Med Mol Imaging. 2018;45(12):2055-61.

76. Pereira Mestre R, Treglia G, Ferrari M, et al. Correlation between PSA kinetics and PSMA-PET in prostate cancer restaging: a metaanalysis. Eur J Clin Investig. 2019;49(3):e13063. https://doi.org/10. 1111/eci.13063.

77. Ceci F, Herrmann K, Hadaschik B, Castellucci P, Fanti S. Therapy assessment in prostate cancer using choline and PSMA PET/CT. Eur J Nucl Med Mol Imaging. 2017;44(Suppl 1):78-83.

78. Fanti S, Hadaschik B, Herrmann K. Proposal of systemic therapy response assessment criteria in time of PSMA PET/CT imaging: PSMA PET Progression (PPP) [published online ahead of print, 2019 Dec 5]. J Nucl Med. 2019:jnumed.119.233817.

79. Hofman MS, Emmett L, Violet J, et al. TheraP: a randomized phase 2 trial of 177 Lu-PSMA-617 theranostic treatment vs cabazitaxel in progressive metastatic castration-resistant prostate cancer (Clinical Trial Protocol ANZUP 1603). BJU Int. 2019;124(Suppl 1):5-13.

80. Thang SP, Violet J, Sandhu S, et al. Poor outcomes for patients with metastatic castration-resistant prostate cancer with low prostatespecific membrane antigen (PSMA) expression deemed ineligible for 177Lu-labelled PSMA radioligand therapy. Eur Urol Oncol. 2019;2(6):670-6. 
81. Hofman MS, Violet J, Hicks RJ, et al. [177Lu]-PSMA-617 radionuclide treatment in patients with metastatic castration-resistant prostate cancer (LuPSMA trial): a single-centre, single-arm, phase 2 study. Lancet Oncol. 2018;19(6):825-33.

82. Violet J, Sandhu S, Iravani A, et al. Long term follow-up and outcomes of re-treatment in an expanded 50 patient single-center phase II prospective trial of Lutetium-177 (177Lu) PSMA-617 theranostics in metastatic castrate-resistant prostate cancer [published online ahead of print, 2019 Nov 15]. J Nucl Med. 2019: jnumed.119.236414.

Publisher's note Springer Nature remains neutral with regard to jurisdictional claims in published maps and institutional affiliations.

\section{Affiliations}

\section{Francesco Ceci ${ }^{1}$ - Daniela E. Oprea-Lager ${ }^{2}$ (D) Louise Emmett $^{3,4}$. Judit A. Adam ${ }^{5}$. Jamshed Bomanji ${ }^{6}$. Johannes Czernin ${ }^{7}$. Matthias Eiber ${ }^{8}$. Uwe Haberkorn ${ }^{9}$. Michael S. Hofman ${ }^{10,11}$ - Thomas A. Hope ${ }^{12}$. Rakesh Kumar ${ }^{13}$. Steven P. Rowe ${ }^{14}$. Sarah M. Schwarzenboeck ${ }^{15}$. Stefano Fanti ${ }^{16}$ - Ken Herrmann ${ }^{17}$}

1 Nuclear Medicine, Department of Medical Sciences, University of Turin, Turin, Italy

2 Department of Radiology \& Nuclear Medicine, Amsterdam University Medical Centers, VU University Medical Center, Cancer Center Amsterdam, De Boelelaan 1117, 1081

HV Amsterdam, The Netherlands

3 St. Vincent's Clinical School, University of New South Wales, Kensington, NSW, Australia

4 Department of Theranostics and Nuclear Medicine, St. Vincent's Hospital Sydney, Darlinghurst, NSW, Australia

5 Department of Radiology \& Nuclear Medicine, Amsterdam University Medical Centers, University of Amsterdam, Amsterdam, The Netherlands

6 Department of Nuclear Medicine, University College London Hospitals NHS Foundation Trust, London, UK

7 Ahmanson Translational Theranostics Division, Department of Molecular and Medical Pharmacology, David Geffen School of Medicine, University of California Los Angeles, Los Angeles, CA 90095, USA

8 School of Medicine, Department of Nuclear Medicine, Technische Universität München, Munich, Germany
9 Department of Nuclear Medicine, Heidelberg University Hospital, Heidelberg, Germany

10 Molecular Imaging and Therapeutic Nuclear Medicine, Peter MacCallum Cancer Centre, Melbourne, VIC, Australia

11 Sir Peter MacCallum Department of Oncology, University of Melbourne, Melbourne, VIC, Australia

12 Department of Radiology and Biomedical Imaging, University of California San Francisco, San Francisco, CA, USA

13 Department of Nuclear Medicine, All India Institute of Medical Sciences, New Delhi, India

14 Division of Nuclear Medicine, The Russell H. Morgan Department of Radiology and Radiological Science, Johns Hopkins University School of Medicine, Baltimore, MD, USA

15 Department of Nuclear Medicine, Rostock University Medical Centre, Rostock, Germany

16 Nuclear Medicine, IRCCS Azienda Ospedaliero-Universitaria di Bologna, University of Bologna, Bologna, Italy

17 Department of Nuclear Medicine, University of Duisburg-Essen and German Cancer Consortium (DKTK), University Hospital Essen, Essen, Germany 